\title{
Wegener granulomatosis of the heart
}

Noriyuki Abe, MD, Hiroaki Takahashi, MD, PhD, Takeshi Inoue, MD, Hiroshi Tanaka, MD, PhD, and Yutaka Okita, MD, PhD, Kobe, Japan

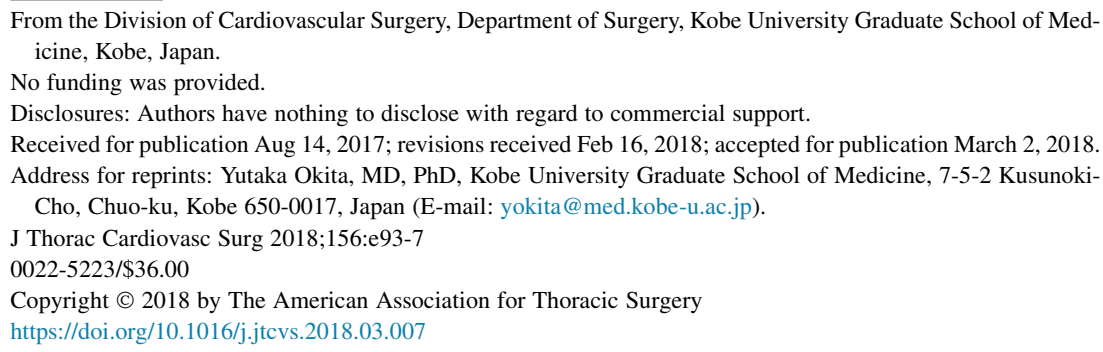

From the Division of Cardiovascular Surgery, Department of Surgery, Kobe University Graduate School of Medicine, Kobe, Japan.

No funding was provided.

Disclosures: Authors have nothing to disclose with regard to commercial support.

Received for publication Aug 14, 2017; revisions received Feb 16, 2018; accepted for publication March 2, 2018.

Address for reprints: Yutaka Okita, MD, PhD, Kobe University Graduate School of Medicine, 7-5-2 Kusunoki-

Cho, Chuo-ku, Kobe 650-0017, Japan (E-mail: yokita@med.kobe-u.ac.jp).

J Thorac Cardiovasc Surg 2018;156:e93-7

$0022-5223 / \$ 36.00$

Copyright (c) 2018 by The American Association for Thoracic Surgery

https://doi.org/10.1016/j.jtcvs.2018.03.007

Video clip is available online.

Wegener granulomatosis (granulomatosis with polyangiitis [GPA]) is a progressive systemic polyangiitis that may involve the respiratory tract or kidneys. Cardiac lesions, however, are rarely reported. We report here 2 cases of GPA in which a central fibrous body was involved. The institutional ethics committee provided appropriate approval.

\section{CLINICAL SUMMARIES}

In case 1, a 55-year-old woman with complete atrioventricular block was referred to our hospital. Physical examination revealed a saddle nose. The result of cytoplasmic anti-neutrophil cytoplasmic antibody testing was positive. An echocardiogram revealed mild aortic valve regurgitation and moderate mitral valve regurgitation. A solid cardiac tumor rapidly expanded (Figure 1, A). Enhanced computed tomography showed infiltration of the cardiac tumor into the aortic root, septal tricuspid leaflet, mitral valve, and aortomitral continuity (Figure 1, B), with the coronary artery remaining intact. Cardiac magnetic resonance imaging revealed an isointense mass on T1weighted images and a mildly hyperintense mass on T2weighted images. A late gadolinium enhancement imaging revealed marked homogeneous enhancement of the mass (Figure 1, C). Fludeoxyglucose F 18 (INN fludeoxyglucose $\left.\left[{ }^{18} \mathrm{~F}\right]\right)$ positron emission tomography demonstrated mild uptake (maximum standardized uptake value, 6.5; Figure 1, D).

In case 2, a 72-year-old woman with cardiac dilatation and complete atrioventricular block was referred to our

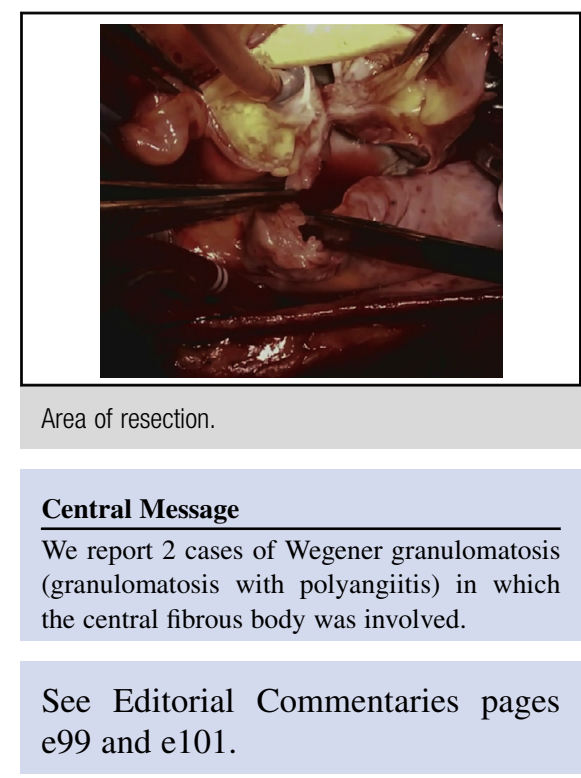

hospital; the patient had torsades de pointes develop, and a cardiac pacemaker was surgically implanted. Echocardiography showed moderate aortic valve regurgitation, mild mitral valve regurgitation, and a dilated left ventricle. The clinical examination showed a result similar to that of case 1 (Figure 2).

\section{Surgical Technique}

Cardiopulmonary bypass was established through a full sternotomy. A thick, fibrous tumor was observed in the Valsalva sinus up to the aortic cusp, as well as in the left atrium, anterior leaflet of the mitral valve, atrial septum, and septal leaflet of the tricuspid valve (Figure 3). The roof of the left atrium was incised through the superior transseptal approach. We used a Manouguian incision to excise the anterior leaflet of the mitral valve, trigone, and aortic root (Figure 1,E).

The mitral valve was replaced with an Epic 27 mm (St Jude Medical, Inc, St Paul, Minn) bioprosthetic valve. The mitral annulus and left atrial roof were reconstructed with a large bovine pericardial patch (Xenomedica; Edwards Lifesciences LLC, Irvine, Calif). The aortic annulus was reconstructed with bovine pericardium. The left ventricular outflow tract was reconstructed with a composite bioprosthesis (Trifecta $23 \mathrm{~mm}$; St Jude Medical) and a 28-mm Valsalva graft (Vascutek, Inchinnan, United 

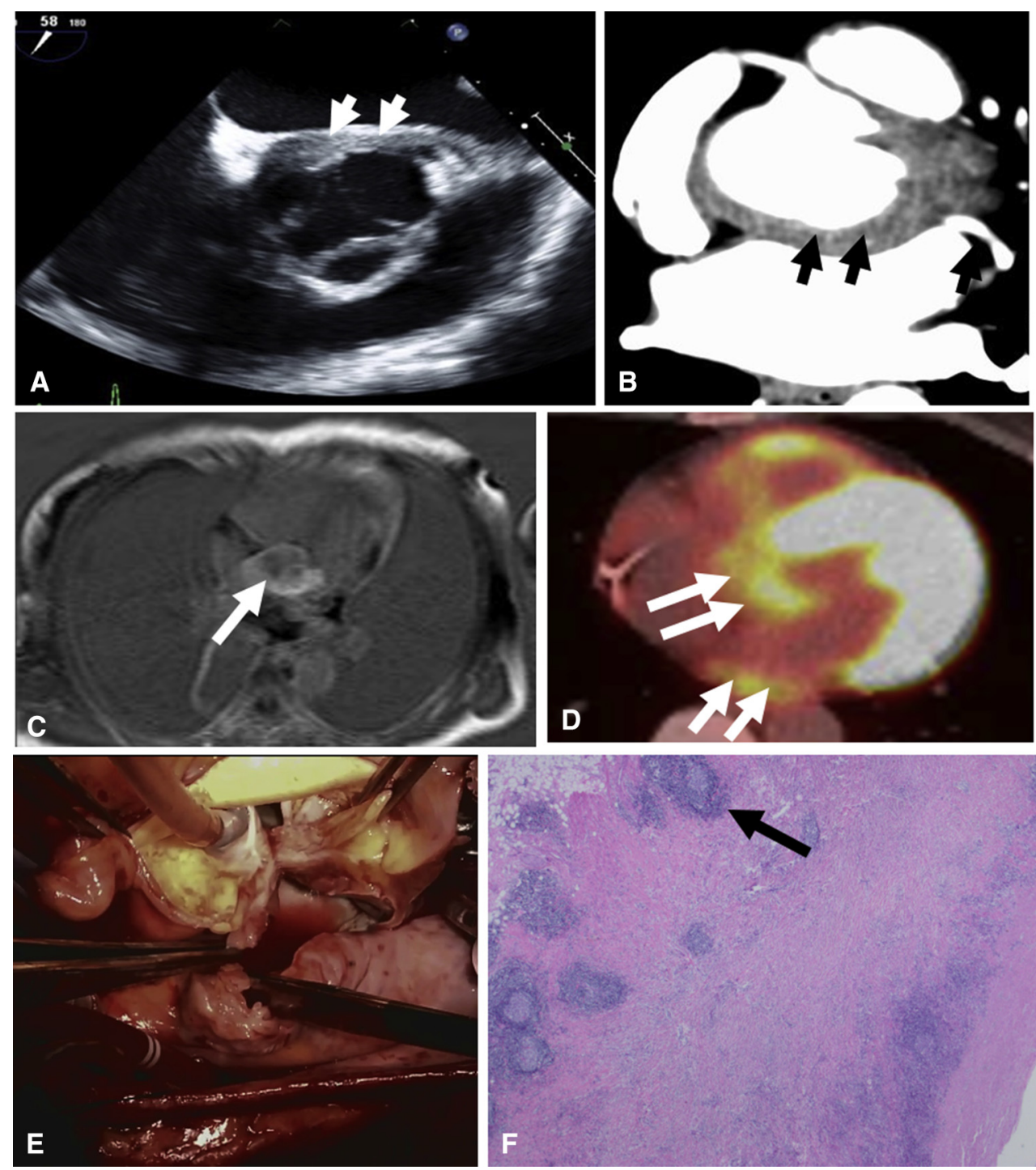

FIGURE 1. Preoperative imaging findings in case 1. A, Transesophageal echocardiography revealed a sessile cardiac tumor (white arrow) abutting the aortic root and extending to the left atrial roof, anterior mitral leaflet, and atrial septum. B, Contrast-enhanced computed tomography showed cardiac tumor (black arrow) infiltrating the aortic root up to the septal tricuspid leaflet, mitral valve, and aortomitral continuity, with no signs of lymphadenopathy. C, Cardiac magnetic resonance imaging (late gadolinium enhancement) revealed marked homogeneous enhancement (white arrow). D, Fludeoxyglucose $\mathrm{F}$ 18 positron emission tomography and computed tomography demonstrated mild to moderate uptake in the aortic root and the anterior mitral leaflet (maximum standardized uptake value, 6.5) and homogeneous uptake (white double arrow) in the left atrium, right ventricular outflow tract, and pulmonary artery. E, Intraoperative photography documented that a Manouguian incision excised the anterior leaflet of the mitral valve, trigone, and aortic root. F, Pathologic findings showed lymphoid follicles (arrow), which were mainly localized to the epicardium.

Kingdom). The coronary buttons were reattached to the composite graft (Video 1).

In the second case, a composite graft reconstruction was also performed (Trifecta $21 \mathrm{~mm}$; St Jude Medical) and Valsalva graft $26 \mathrm{~mm}$ (Vascutek), mitral valve replacement was performed with an Epic $27 \mathrm{~mm}$ (St Jude Medical), and tricuspid valve annuloplasty was performed with a Contour $28 \mathrm{~mm}$ (Medtronic Inc, Minneapolis, Minn). The central fibrous body was reconstructed with the same method. Rapid pathologic examination showed inflammation rather than a malignancy.

\section{Pathologic Findings (Figure 1, F)}

The mass was consistent with autoimmune disease. It was highly suggestive of GPA because of intense chronic necrotizing granuloma, chronic intense lymphoplasmacytic 


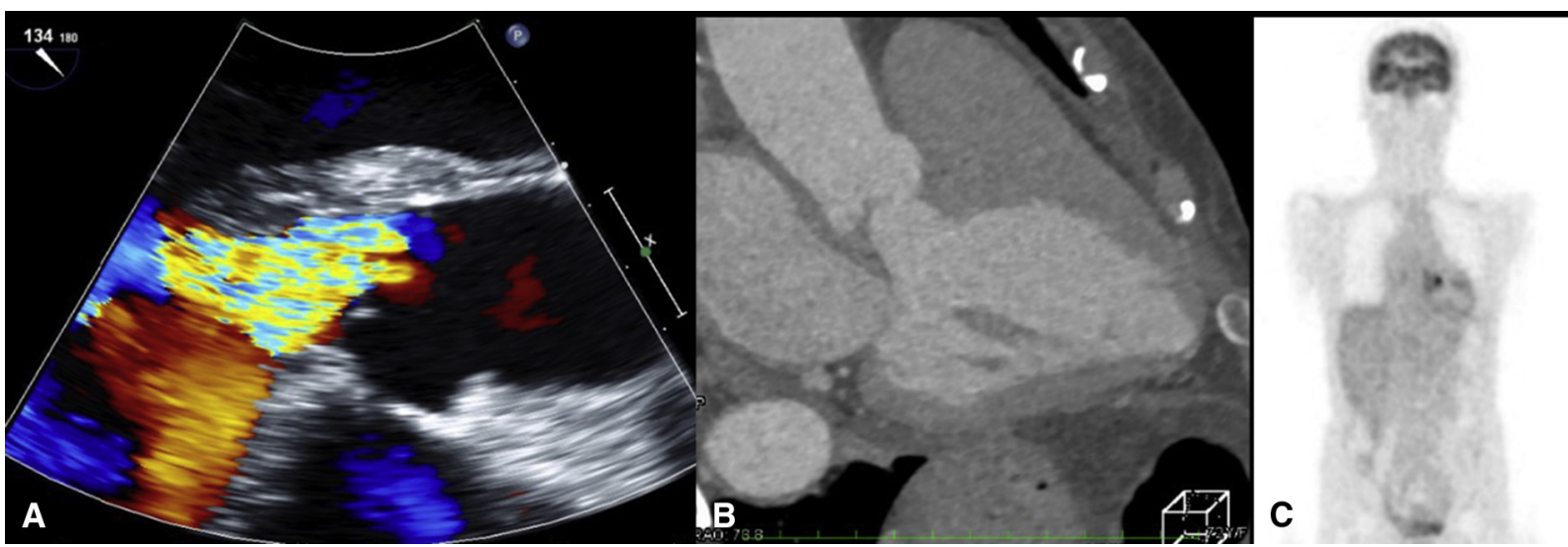

FIGURE 2. Preoperative imaging findings in case 2. A, Transesophageal echocardiography revealed moderate aortic valve regurgitation with a sessile cardiac tumor extending to the aortic root. B, Contrast-enhanced computed tomography showed a cardiac tumor infiltrating the aortic root up to the mitral valve and aortomitral continuity. C, Fludeoxyglucose F 18 positron emission tomography demonstrated a mild uptake to the anterior mitral leaflet (maximum standardized uptake value, 4.5$)$.

inflammation, fibrosis with hyalinization, and neutrophilic microabscesses involving the central fibrous body (Figure 4).

\section{Postoperative Course}

Both patients had a straightforward postoperative course and were discharged. At 9 and 7 months postoperatively, both were reportedly living normal lives, without the need for steroids.

\section{Additional Cases}

In case 3, a 79-year-old man was referred to our hospital for subacute type A aortic dissection. He had a history of Wegener granulomatosis and rapidly progressive glomerulonephritis. He had undergone a double valve replacement for active infectious endocarditis 10 years previously. We performed a hemiarch replacement and reoperative mitral valve replacement for structural valve deterioration and a type A aortic dissection (Table 1).

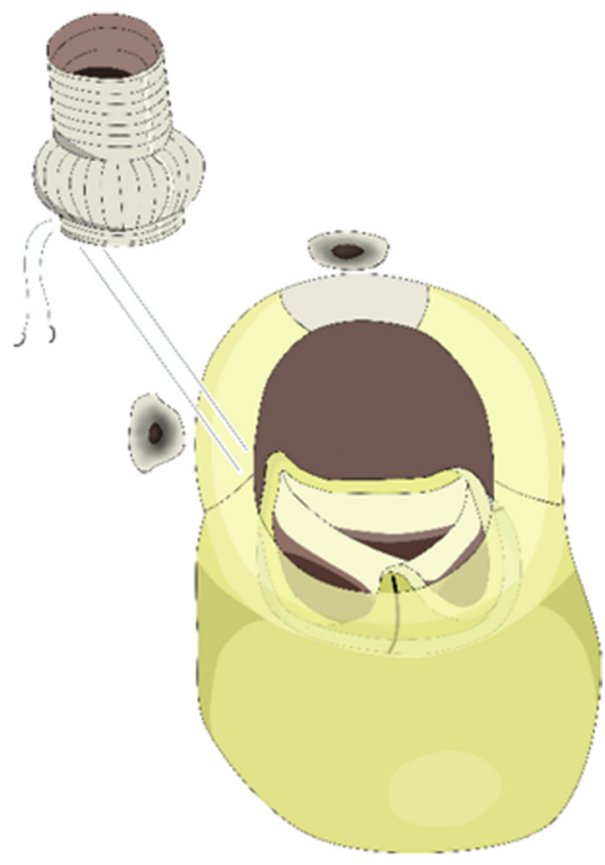

FIGURE 3. Surgical technique. A, During reconstruction, the prosthetic Epic $27 \mathrm{~mm}$; (St Jude Medical, Inc, St Paul, Minn) mitral valve was seated on the preserved posterior mitral valve annulus. The annuli of the anterior side and fibrous trigone were covered with a large bovine pericardial graft, which was used to roof the left atrium. B, The stented prosthetic valve consisted of a 23-mm Trifecta (St Jude Medical) and 28-mm Valsalva graft (Vascutek, Inchinnan, United Kingdom), which was seated on the neoaortic annulus. 


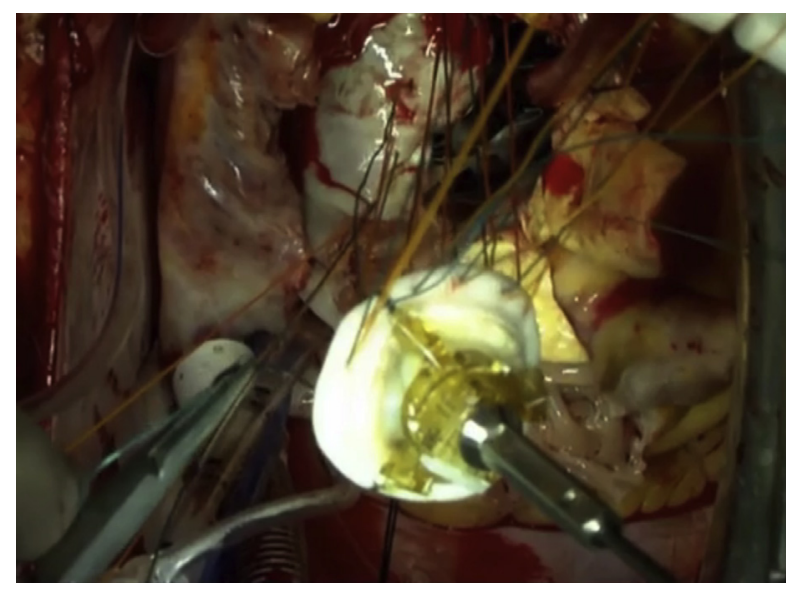

VIDEO 1. A thick, fibrous tumor was observed from the Valsalva sinus to the aortic cusp, left atrium, anterior leaflet of the mitral valve, atrial septum, and septal leaflet of the tricuspid valve. Through the superior transseptal approach, the roof of left atrium was incised. We used a Manouguian incision to excise the anterior leaflet of the mitral valve, trigone, and aortic root. The mitral valve was replaced with an Epic $27 \mathrm{~mm}$ (St Jude Medical, Inc, St Paul, Minn) bioprostheses. The mitral annulus and left atrial roof were reconstructed with a large bovine pericardium patch (Xenomedica; Edwards Lifesciences LLC, Irvine, Calif). The aortic annulus was reconstructed with bovine pericardium. The left ventricular outflow tract was reconstructed with a composite bioprosthesis (Trifecta $23 \mathrm{~mm}$; St Jude Medical, Inc) and a 28-mm Valsalva (Vascutek, Inchinnan, Scotland, United Kingdom) graft. The coronary buttons were reattached to the composite graft. Video available at: https://www.jtcvs.org/article/S0022-5223(18)30697-4/fulltext.
In case 4, a 66-year-old man was referred to our hospital for a fever of unknown origin. Echocardiography revealed thick aortic root wall and severe aortic valve regurgitation. We operated with the Bentall procedure. The reconstruction of the coronary button was difficult because of severe inflammation (Table 1).

\section{DISCUSSION}

In the previous literature on Wegener granulomatosis, ${ }^{1} 20$ cases were reported. GPA of the heart was found in $8 \%$, and valvular involvement was seen in $21 \%$ (aortic valve in $57.1 \%$ and mitral valve in $14.3 \%)$.

The histopathologic examination indicated valvular disease. Because the GPA was rapidly progressing, ${ }^{2}$ medical treatment, such as corticosteroids and cyclophosphamide, was recommended for rhythm disorder and coronary vasculitis ${ }^{3,4}$; however, the valvular disease was unclear. In both cases, echocardiography showed aortic valve regurgitation and rapid mitral valve progression. In addition, the tumor was rapidly expanding. The preoperative differential diagnosis included malignant lymphoma, sarcoma, sarcoidosis, and inflammatory diseases. Diagnosis with histopathologic examination required a surgical approach, because the lesion was not amenable to biopsy. We therefore considered radical resection of the tumor.

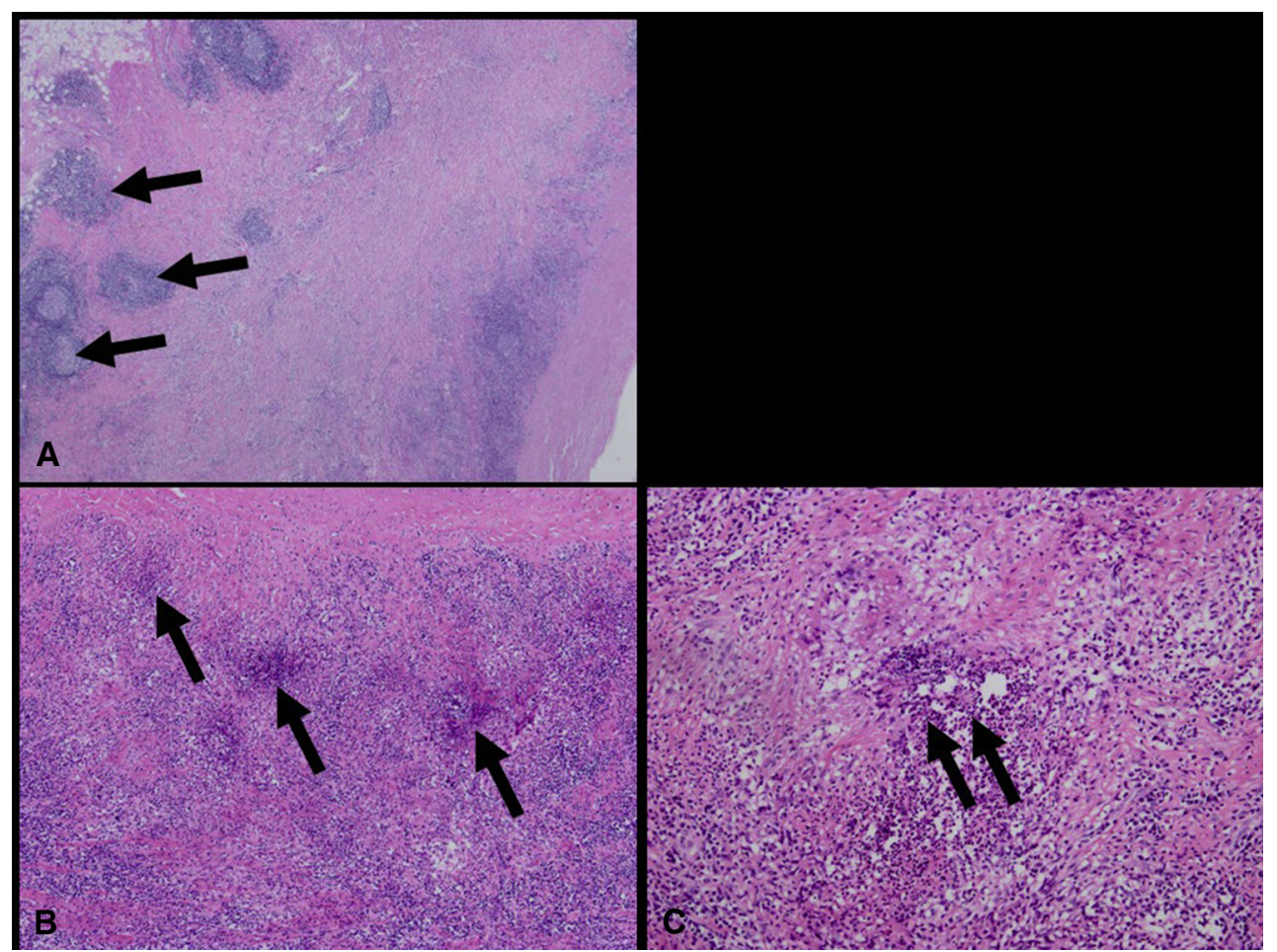

FIGURE 4. Pathologic findings: A, Panel shows lymphoid follicles (arrows) which were mainly localized to the epicardium. B, Panel shows dirty necrosis and neutrophilic microabscess (arrows) involved in the central fibrous body. C, Panel shows necrotizing granuloma with multinucleated giant cells (double arrows). 
TABLE 1. Clinical summary

\begin{tabular}{lcclc}
\hline Case & Age $(\mathbf{y})$ & Sex & \multicolumn{1}{c}{ Lesions } & Surgery \\
\hline 1 & 55 & F & AR; MR; thickening of aortic wall & Bentall plus MVR \\
2 & 72 & F & AR; MR; thickening of aortic wall & Bentall plus MVR \\
3 & 79 & M & Aortic dissection; thickening of aortic wall; SVD & Redo MVR; hemiarch \\
4 & 66 & M & AR; thickening of aortic wall & Bentall \\
\hline
\end{tabular}

$A R$, Aortic valve regurgitation; $M R$, mitral valve regurgitation; $M V R$, mitral valve replacement; $S V D$, structural valve deterioration.

The surgical strategy reported by Nilto and colleagues ${ }^{5}$ with the Manouguian incision and Bentall procedure was useful. They were able to resect both the aortic and mitral annuli for infectious tissue or bulky calcification in the area between the 2 trigones. We radically resected the central fibrous body tumor with this approach.

\section{CONCLUSIONS}

This report highlights 2 rare cases of Wegener granulomatosis (granulomatosis with polyangiitis) on the central fibrous body of the heart.

\section{References}

1. Lacoste C, Mansencal N, Ben M'rad M, Goulon-Goeau C, Cohen P, Guillevin L, et al. Valvular involvement in ANCA-associated systemic vasculitis: a case report and literature review. BMC Musuloskelet Disord. 2011;12:50.

2. Al-Kindi SG, Amer Al-Aiti M, Yang M, Josephson RA. Granulomatosis with polyangiitis presenting with acute aortic and mitral regurgitation: case report and bigdata analysis. J Heart Valve Dis. 2017;26:103-6.

3. Raghunathan V, Pelcovits A, Gutman D, Carino G. Cardiogenic shock from coronary vasculitis in granulomatosis with polyangiitis. BMJ Case Rep. 2017; https://doi.org/10.1136/bcr-2017-220233.

4. Schiavone WA, Ahmad M, Ockner SA. Unusual cardiac complications of Wegener's granulomatosis. Chest. 1985;88:745-8.

5. De Oliveira NC, David TE, Armstrong S, Ivanov J. Aortic and mitral valve replacement with reconstruction of the intervalvular fibrous body: An analysis of clinical outcomes. J Thorac Cardiovasc Surg. 2005;129:286-90. 